\title{
SCADA based wind turbine anomaly detection using Gaussian Process (GP) models for wind turbine condition monitoring purposes
}

\author{
Ravi Kumar Pandit, David Infield \\ Electronics and Electrical Engineering department, University of Strathclyde, 16 Richmond St, \\ Glasgow - G1 1XQ, Scotland, UK. \\ Corresponding author Email: ravi.pandit@strath.ac.uk
}

\begin{abstract}
The penetration of wind energy into power systems is steadily increasing; this highlights the importance of operations and maintenance, and also specifically the role of condition monitoring. Wind turbine power curves based on SCADA data provide a cost-effective approach to wind turbine health monitoring.

This paper proposes a Gaussian Process (a non-parametric machine learning approach) based algorithm for condition monitoring. The standard IEC binned power curve together with individual bin probability distributions can be used to identify operational anomalies. The IEC approach can also be modified to create a form of real-time power curve. Both of these approaches will be compared with a Gaussian Process model to assess both speed and accuracy of anomaly detection.

Significant yaw misalignment, reflecting a yaw control error or fault, results in a loss of power. Such a fault is quite common and early detection is important to prevent loss of power generation. Yaw control error provides a useful case study to demonstrate the effectiveness of the proposed algorithms and allows the advantages and limitations of the proposed methods to be determined.
\end{abstract}

\section{Introduction}

The primary role of wind turbine power curves is to provide a benchmark for performance for use in purchase contracts. The measurements and data analysis procedures have been developed over decades and are represented in an International Standard, IEC [1]. There remain issues of accuracy and it is recognised that changes in external factors such as atmospheric stability, and associated with this wind shear and turbulence, can influence the power curves in ways that are not fully understood [1,2,3]. Even air density correction as prescribed in the Standard is imperfect. For example, the authors of [4] used two different windfarm SCADA datasets for constructing power curves using a Gaussian Process and for one of these datasets it was found that model accuracy was marginally improved by avoiding the standard air density pre-correction. Research is needed to further investigate these issues.

In recent years, the potential use of power curves for wind turbine condition monitoring has been recognised $[5,6]$. Significant changes in the power curve can be an indication of developing faults and since SCADA data for operational wind turbines is readily available, techniques for the identification of small changes to the power curve are being researched. As part of this, some researchers have applied new methods to fitting power curves to the data. In most cases the data itself is collected in the same way: 10 minute averaged pairs of net wind turbine power, and hub height horizontal wind speed at a suitable distance in front of the rotor (between 2 and 4 rotor diameters in the Standard), or alternatively taken from the nacelle anemometer.

As mentioned above, power curves are conventionally calculated using the method prescribed by the IEC Standard. This involves binning the data into $0.5 \mathrm{~m} / \mathrm{s}$ wide wind speed intervals, and then calculating the average power and wind speed for each bin. The power curve is a smooth curve drawn through these points, but in actuality is only defined exactly at the points themselves. The bin width of $0.5 \mathrm{~m} / \mathrm{s}$ is a compromise between accuracy, since within each bin the measured power will depend strongly and non-linearly on wind speed and a wide bin would result in a systematic bias, and the need in practice to get sufficient data points in each bin to be of statistical significance. Many papers have been published that seek an alternative approach to fitting power curves to the data. These fall broadly into two categories: parametric methods such a polynomial curve fitting; and nonparametric methods, often using machine learning techniques [7]. These will be briefly reviewed below.

Parametric curve fits to power curves are approximations, with higher order fits generally providing more accurate fitting. Different polynomial curves and an exponential fit are compared in [8]. In [9], improvements on standard least squares polynomial regression using locally weighted polynomial regression and spline regression are demonstrated. It is not immediately clear why these approaches would be considered more attractive than conventional power curves, suitably interpolated.

Kernel methods are non-parametric and a supervised learning form of pattern recognition related to Gaussian Processes, they have been applied to wind turbine power curves. In [10] data from two turbines were analysed and the identification of blade icing was demonstrated. The same authors have also explored an approach based on eigenvalue analysis, [11]. A kernel plus method has also been applied, [12], to overcome identified shortcomings of multivariate kernel regression. Air density, turbulence intensity and wind shear are the additional variables considered. Similar data has been analysed by the same team using the more conventional additive multivariate kernel method, [13].

A somewhat inelegant linear hinge model in which the power curve is broken up into $\mathrm{k}$ segments each fitted using 
This paper is a post-print of a paper submitted to and accepted for publication in IET Renewable Power Generation and is subject to Institution of Engineering and Technology. The copy of record is available at IET Digital Library.

linear regression has been applied, [14]. Clustering approaches have also been used to group data and thus determine a power curve. One example is [15] where a simple cluster centre algorithm is compared with fuzzy Cmeans and subtractive clustering; 8 clusters were found to provide an adequate model. A $\mathrm{k}$ nearest neighbour (k-NN) clustering approach was shown to be superior to a range of other methods including fitting the logistic function through maximum likelihood values, neural nets, random forest and boosting tree. However, the number of clusters used was 150 which is more than the number of bins used in a conventional power curve, so the advantage is unclear.

Many of the published methods concentrate solely on the power curve, but clearly there is valuable information in the spread of data, for example the probability distribution of power points in a given wind speed bin of a conventional power curve. Both Gaussian Process theory and Copula models intrinsically provide confidence bounds for the fitted power curve and these are dealt with in more detail below.

Copula models provide an effective means of representing a non-linear relationship between two variables; they can thus be applied to wind turbine power curves. An early paper, [16], outlines how Copulas can be fitted to wind turbine power curve data. In [17], Copula fitting has been used to exclude outliers when calculating power curves.

Gaussian Processes are very general non-linear models. They have recently started to find application to wind turbine power curves, for example [18]. In Gaussian Process models, training is undertaken to select the best hyper parameters which define the covariance function. The form of the covariance function is commonly assumed to be a square exponential function. In [19], Gaussian Processes are used to model power curves and also to include air density changes. This was found to be preferable to correcting the power-wind speed data to reflect air density as described in the IEC Standard.

This paper proposes a range of algorithms for condition monitoring based on a probabilistic GP and binning methods. A comparative assessment of promising approaches has been undertaken in terms of their capability to detect in advance (and by how much) signs of failure.

\section{Yaw error case study}

All The direction of wind is not constant, it always changes. A wind turbine generates most effectively when the rotor is facing directly into the free oncoming wind; changes in wind direction are tracked by the yaw drive and control system. A wind vane, located on the nacelle behind the rotor blades (figure 1), is used to control the alignment of the wind turbine. Nevertheless, an erroneous yaw error signal can result from turbulence generated with the passage of the blades in spite of sophisticated corrective software, and this will result in a degree of so called yaw error where the turbine rotor is not properly aligned. The yaw error is measured by the wind vane mounted on the rear of the nacelle.

In addition to the challenge of measuring wind direction on the nacelle any misalignment of wind vane itself will contribute to yaw error. Moreover, large wind turbine rotors must be yawed slowly to limit gyroscopic loads and thus cannot follow rapid changes in wind direction. All this means that a level of yaw error in inevitable. This average yaw misalignment will result is some reduction to the annual energy production (AEP). As reported in [21] an average of $6.2^{\circ}$ of misalignment causes an estimated $2 \%$ reduction in AEP with roughly a loss of 2 to $3 \%$ for $9^{\circ}$ to $10^{\circ}$ of average yaw error. As well as the operational yaw error outlined above, faults can develop with the yaw control system. Early detection of such yaw control faults is important to avoid loss of power production and associated revenue [22], but also to minimise fatigue damage and reduce maintenance costs, [23], increase life of turbine, [24], and reduce the levelised Cost of Electricity (LCOE) and improve the return on investment (ROI), [25].

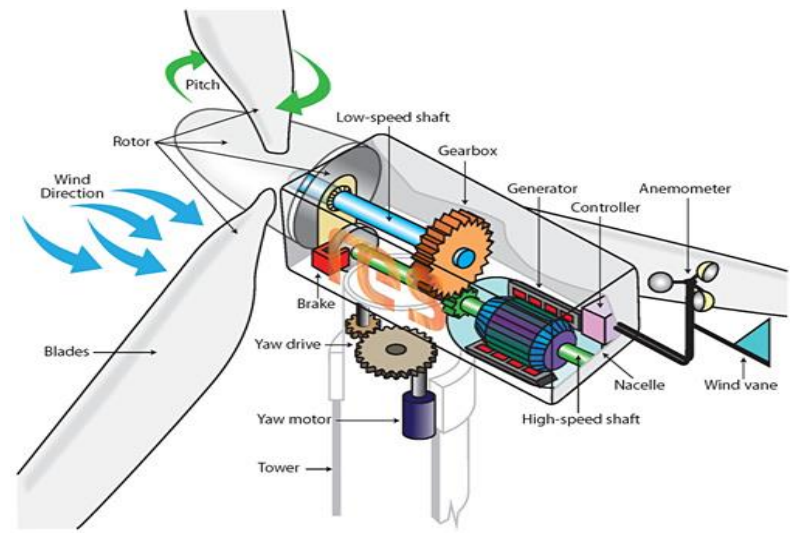

Figure 1: Schematic of wind turbine system, [20]

The dependence of wind turbine power production on yaw error is reasonably explained by cosine cubed theory, [26], which states that power output is scaled by the cube of cosine of yaw error (differences between wind direction and the nacelle direction). Although not perfect, this law estimates that a large yaw error of $20^{\circ}$ will lead to a significant power deficit of $17 \%$ Such power deficit is unlikely to acceptable to a wind farm operator. Because of the strong link between yaw error and power production, such faults can provide excellent test cases with which to test power curve anomaly detection algorithms.

\section{Data description and pre-processing}

There is considerable pressure to reduce the cost of wind energy, especially from offshore installations. Condition monitoring has the potential to assist in this, but many condition monitoring systems are expensive and require an experienced engineer to interpret the data. Condition monitoring using Supervisory Control and Data Acquisition (SCADA) data is a cost effective approach since such data is available at no cost. SCADA data provides crucial knowledge regarding load history and operations of individual wind turbines and provides an efficient and costeffective way to monitor wind turbines for early warning of failures and related performance issues.

The SCADA data sets used in this study are from operational wind turbines located in Scotland, UK, and contain more than 100 different signals, ranging from timestamp, calculated values, set point, measurements of 
This paper is a post-print of a paper submitted to and accepted for publication in IET Renewable Power Generation and is subject to Institution of Engineering and Technology. The copy of record is available at IET Digital Library.

temperature, current, voltage, wind speed, power output, wind direction etc.

Due to sensor failure and data collection faults SCADA data is not itself without errors. Such errors will affect power curves and should be systematically removed at the outset. Criteria like timestamp mismatches, out of range values, negative power values, and turbine power curtailment are considered to be misleading and have been filtered out following methodologies used in [27] and summarized in table 1. All the SCADA data considered here consists of 10 minute averages with maximum, minimum, and standard deviation over the 10 minutes also being recorded; the available data set corresponds to a full year of operation. The data used in this paper is for 2.3 MW Siemens turbines. Table 1 summarises two datasets: data set 1 beginning with time stamp " $11 / 1 / 2008$ 14:30 PM" and ending at time stamp “'30/03/2008 15:20 PM'; and data set 2 beginning with time stamp " $14 / 4 / 2009$ 11:20 AM' and ending at time stamp “'16/4/2009 9:50 PM'. Data set 1 contains 4725 measured

\begin{tabular}{cccc}
\hline $\begin{array}{c}\text { Data } \\
\text { set }\end{array}$ & $\begin{array}{c}\text { Start time } \\
\text { stamp }\end{array}$ & $\begin{array}{c}\text { End time } \\
\text { stamp }\end{array}$ & Description \\
\hline $\mathbf{1}$ & $11 / 1 / 2008$ & $30 / 03 / 2008$ & Total data \\
& $14: 30 \mathrm{PM}$ & $15: 20 \mathrm{PM}$ & filtered set:
\end{tabular}

observations

$\begin{array}{cccc}2 & 14 / 4 / 2009 & 16 / 4 / 2009 & \text { Total data set: } \\ & 11: 20 \text { AM } & 9: 50 \text { AM } & 201 \text { observations }\end{array}$

value which became 3274 data points after pre-processing and was used to develop a power curve models based on binning and a Gaussian Process (GP), as described in upcoming sections. Data set 2 includes 201 data points and was used to test the performance of the model learned from data set 1 .

Table 1: Description of the data sets

\section{Construction of reference power curve and importance of air density correction}

The nonlinear relationship between hub wind speed and turbine output power is represented by the power curve; mathematically it is described by the following equation,

$$
P=0.5 \rho A C_{p}(\lambda, \beta) v^{3}
$$

where, $\rho$ is Air density $\left(\mathrm{kg} / \mathrm{m}^{3}\right)$, A is swept area $\left(\mathrm{m}^{2}\right)$, $C_{p}$ is the power coefficient of wind turbine and $v$ is the hub wind speed $(\mathrm{m} / \mathrm{sec})$.

The power coefficient is a function of tip speed ratio $(\lambda)$ and pitch angle $(\beta)$. In addition to these two parameters, the power capture from a wind turbine depends on other factors such as wind direction, wind shear, turbulence intensity and air density [28]. Due to the complex manner in which these parameters impact on turbine efficiency, equation (1) must be regarded as a simplification with limited accuracy.

A key application of the power curve is in the estimation of annual energy production (AEP), calculated from the wind speed frequency distribution and the corresponding power values given by the power curve. Wind resource assessment of a site or region is undertaken using the long term probability distribution of hub wind speed together with the power curve, [29]. The Capacity factor (CF), defined as the ratio of actual energy production to production if the turbine operated always at rated power, is used to assess how effective a turbine is at a given location. CF can be used to determine optimum turbine-site matching and rank potential sites [30,31]. In [32], four different power curve models were applied in the estimation of capacity factor. Accurate modelling of a wind turbine power curve can assist in the analysis of turbine performance and efficiency and thus in anomaly detection $[33,34]$.

Air density changes with passing weather systems, altitude and significantly with ambient temperature. Hence in order to analyse the performance of modern variable speed turbines, an air density correction should be applied as per IEC standard 61400-12-1, [1,35] and is given as follows,

$$
\rho=1.225\left[\frac{288.15}{T}\right]\left[\frac{B}{1013.3}\right]
$$

and,

$$
V_{C}=V_{M}\left[\frac{\rho}{1.225}\right]^{\frac{1}{3}}
$$

where, $V_{C}$ and $V_{M}$ are the corrected and measured wind speed in $\mathrm{m} / \mathrm{sec}$ and the corrected air density is calculated by equation (2) where B is atmospheric pressure in mbar and $\mathrm{T}$ the temperature in Kelvin. It should be noted $\mathrm{T}$ and $\mathrm{B}$ values are 10 - minute average values extracted from SCADA data. The corrected wind speed $\left(V_{C}\right)$ from equation (3) is then used to calculate the power curve.

10-minute SCADA data from a healthy turbine are utilized for reference power curve construction. The power curve data shown in figure 2 is after the correction for air density and after removing misleading data as described in section 3 . These air density corrections also applied to the unhealthy turbine data so that it can be evaluated against the reference power curve in a consistent manner.

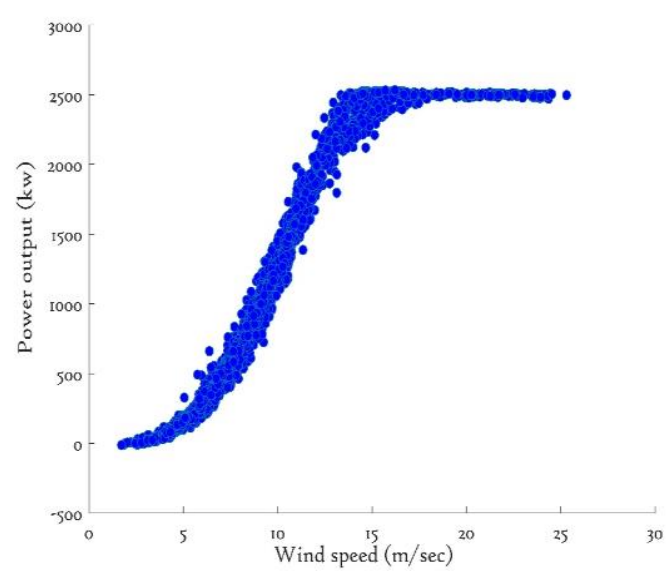

Figure 2: Pre-processed power curve and affiliations 
This paper is a post-print of a paper submitted to and accepted for publication in IET Renewable Power Generation and is subject to Institution of Engineering and Technology. The copy of record is available at IET Digital Library.

SCADA data from the wind turbine comes with measurement errors so it is desirable to add a noise term to

\section{Basics of Gaussian Process (GP)}

An A Gaussian Process (GP) is a Bayesian, nonparametric, non-linear regression machine learning approach widely used to deal with probabilistic regression problems, for example, [36,37]. Because of its flexibility, its basis in probability theory and ease of modelling (it requires very few assumptions) it is an ideal approach for many prediction and fitting related issues. A GP is in essence the non-parametric generalization of a joint normal distribution for a given potentially infinite set of variables and it is mathematically defined by its mean and covariance functions (or kernel) as given in equation (4),

$$
\mathrm{Y} \sim \mathrm{GP}(\mu, \Sigma)
$$

where, $\mu$ is the mean function, and $\sum$ is the covariance function that has an associated probability density function:

$$
P(x ; \mu, \Sigma)=\frac{1}{(2 \pi)^{\frac{n}{2}}|\Sigma|^{\frac{1}{2}}} \exp \left\{-\frac{1}{2}(x-\mu)^{T} \Sigma^{-1}(x-\mu)\right\}
$$

where $\left|\sum\right|$ is defined as determinant of $\sum, n$ is the dimension of random input vector $x$, and $\mu$ is mean vector of vector $x$. The term under the exponential, i.e. $\frac{1}{2}(x-$ $\mu)^{T} \Sigma^{-1}(x-\mu)$ is an example of a quadratic form.

The 1-dimensional Gaussian Process model is a special case where $x$ is a scalar. The covariance matrix, $K$, gives the variance of each variable along the leading diagonal, and the off-diagonal elements measure the correlations between the different variables, and are given by:

$$
K=\left[\begin{array}{ccc}
k_{11} & \cdots & k_{1 n} \\
\vdots & \ddots & \vdots \\
k_{n 1} & \cdots & k_{n}
\end{array}\right] \text { where } k_{i j}=k\left(x_{i}, x_{j}\right)
$$

$K$ is of size $n \times n$, where $n$ is the number of input parameters considered, and it must be symmetric and positive semidefinite i.e. $\sum_{i j}=\sum_{j i}$.

A covariance function describes the dependency of two variables with respect to each other and is the heart of any GP model; it signifies the similarity between two points and hence determines closeness between two points. GP model behaviour is completely defined by its co-variance functions which makes selection of suitable covariance function in a GP model necessary for prediction model accuracy.

There are various available covariance functions described in [37] and selection is based on nature of the data. The squared exponential covariance function is commonly applied and will be used in this paper. For any finite collection of inputs $\{x 1, x 2, \ldots \ldots, x n\}$, it is defined as:

$$
k_{S E}\left(x, x^{\prime}\right)=\sigma_{f}^{2} \exp \left(-\frac{\left(x-x^{\prime}\right)^{2}}{2 l^{2}}\right)
$$

the covariance function in order to improve the accuracy of the GP model. Hence equation (6) modified to be:

$$
k_{S E}\left(x, x^{\prime}\right)=\sigma_{f}^{2} \exp \left(-\frac{\left(x-x^{\prime}\right)^{2}}{2 l^{2}}\right)+\sigma_{n}^{2} \delta\left(x, x^{\prime}\right)
$$

where $\sigma_{f}^{2}$ and $l$ are known as the hyper-parameters. $\sigma_{f}^{2}$ Signifies the signal variance and $l$ is a characteristic length scale which describes how quickly the covariance decreases with distance between points. $\sigma_{n}$ is the standard deviation of the noise fluctuation and gives information about model uncertainty. $\delta$ is the Kronecker delta, [37].

Using this GP model, a power curve is estimated from the data and is shown in figure 3 . As can be seen clearly from figures 4 and 5, this GP model is able to fit the power curve smoothly and accurately.

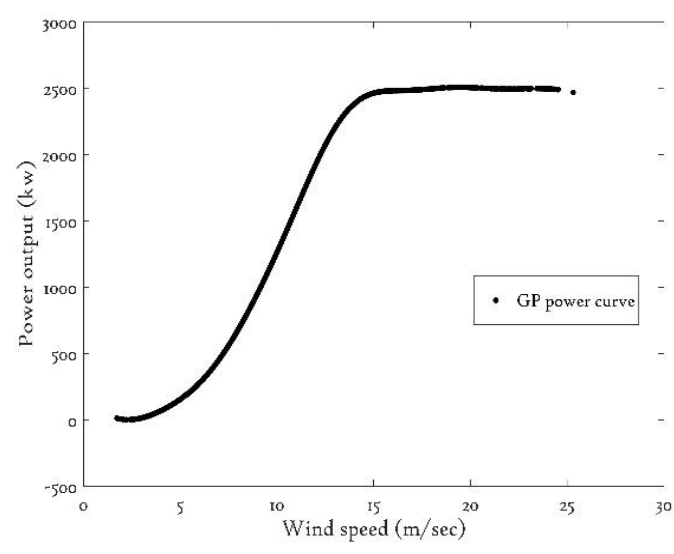

Figure 3: GP fitted power curve

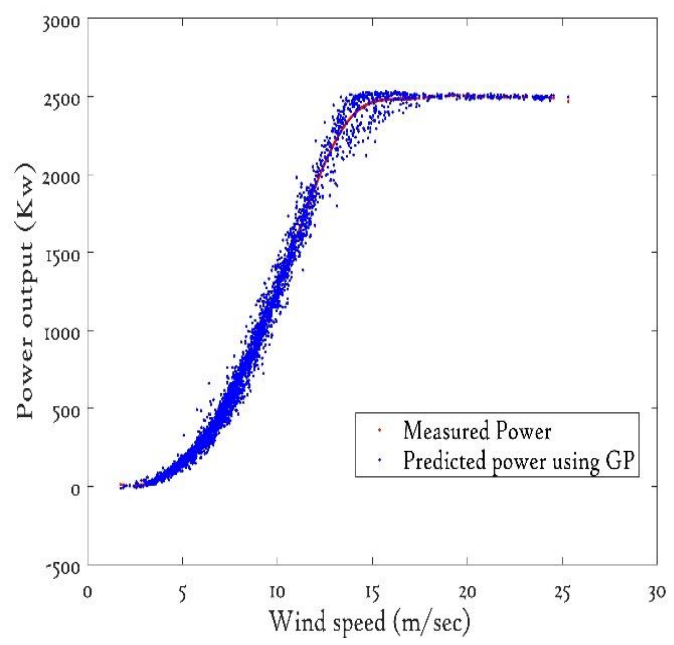

Figure 4: GP power curve comparison 
This paper is a post-print of a paper submitted to and accepted for publication in IET Renewable Power Generation and is subject to Institution of Engineering and Technology. The copy of record is available at IET Digital Library.

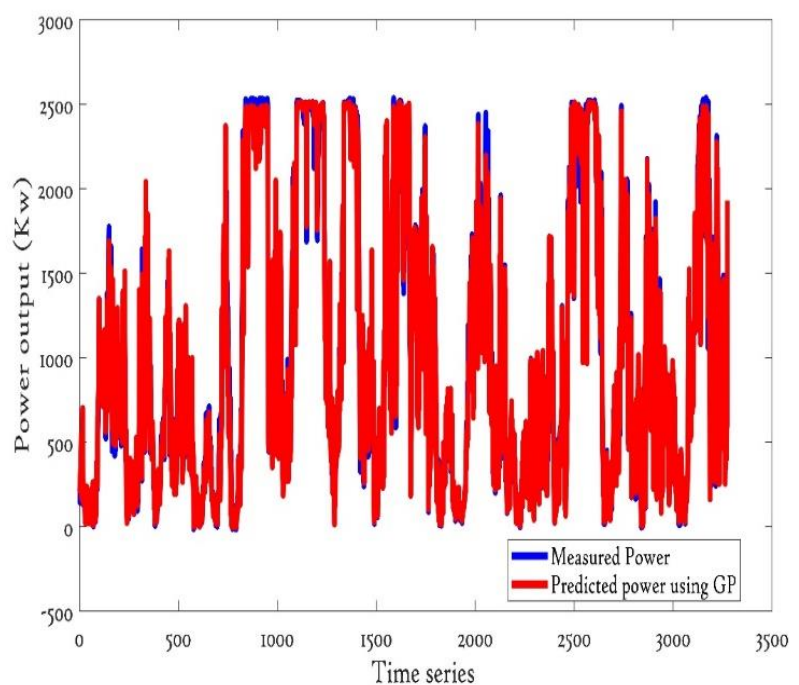

Figure 5: GP estimates of power generation time series
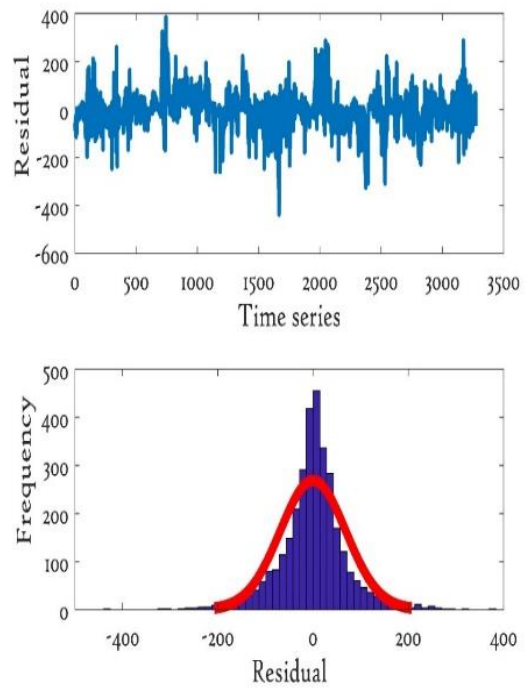

Figure 6: Plot of residuals and its histogram fitting

Investigation of residuals of a GP model is important. The frequency distribution of the residuals is shown in figure 6 together with a fitted Gaussian distribution. This distribution validation is useful for developing the probabilistic based GP model in section 6.1.3.

\section{Methodologies to be compared}

Wind turbine performance analysis is presently an active area of research. This paper is focused on performance as represented by fitted power curves.

\subsection{Power curve comparison}

Power curves can play a vital role in identifying anomalous operation reflecting insipient fault development. Information related to statistically significant power curve deviations is thus important. Such deviations could be due to: anemometer error, power transducer calibration error or controller setting error. A consistent significant deviation from the reference power curve may be due to blade damage; yaw drive issues or possibly very large wind shear or wind veer for below rated operation, [38]). The IEC recommended approach uses binning; hence in order to evaluate the effectiveness of the GP model, two binning methods are described and a comparative analysis carried out.

In this paper, based on a reference power curve constructed using either binning or a GP model, as outlined above, an anomaly detection algorithm has been constructed to identify any statistically significant deviation from the reference power curve on bin by bin basis for the binned reference power curve, and for the GP model using the inherent confidence intervals.

\subsubsection{Fisher's combined probability test}

Statistical tools often need to combine the evidence obtained from assumed independent sources. In order to do so, a combine p-values concept is developed. The Fisher product test, [39], is a statistical test that combines p-values, based on notion that several non-significant results occurring together may suggest significance and hence detect departure from the null hypothesis , $H_{o}$. The equation used for calculating the Fisher combined probability test is given as,

$$
X_{2 k}^{2} \sim-2 \sum_{i=1}^{k} \ln p_{i}
$$

where $\mathrm{p}_{\mathrm{i}}$ is the probability that the $i^{\text {th }}$ variable exceeds the measured value under the null hypothesis . Under the null hypothesis $\mathrm{X}_{2 \mathrm{k}}^{2}$ is the distributed as a chi-squared variate with $2 k$ degrees of freedom. Here, $k$ is the number of independent tests being performed.

Apart from Fisher method, other methods for combining p-values are briefly discussed in [40, 41,42]. The applications for combining the p-values are numerous, including combining the results from independent studies and combining the results of individual component problems as part of an overall test, [43, 44]. This approach will be used to construct effective probabilistic based binning and for the GP algorithm, as is described in the following sections.

For consistency, all the time series used in this work covers the same time period of 33 hours. On the graphs these are shown on a scale of zero to 200 (10-minute periods); in reality the time series runs from 11:20 on 14-04-2009.

\subsubsection{Probabilistic assessment of incoming data using a binned power curve}

This algorithm uses a probabilistic approach to identify anomalies associated with incoming power-wind speed data, point by point. A reference binned power curve has been constructed following the IEC recommendations using the pre-processed power curve (of figure 2) where it is binned by the normal $0.5 \mathrm{~m} / \mathrm{sec}$ wide wind speed intervals. Figure 7 shows the reference binned power curve together with error bars. The two standard deviation (i.e. 95\% confidence intervals) of measured power values are used to calculate the error bars which are used to measure the uncertainty associated with each bin of the power curve. It is worth noting that there is slight deviation in power curve which is due to varying environmental conditions. 
This paper is a post-print of a paper submitted to and accepted for publication in IET Renewable Power Generation and is subject to Institution of Engineering and Technology. The copy of record is available at IET Digital Library.

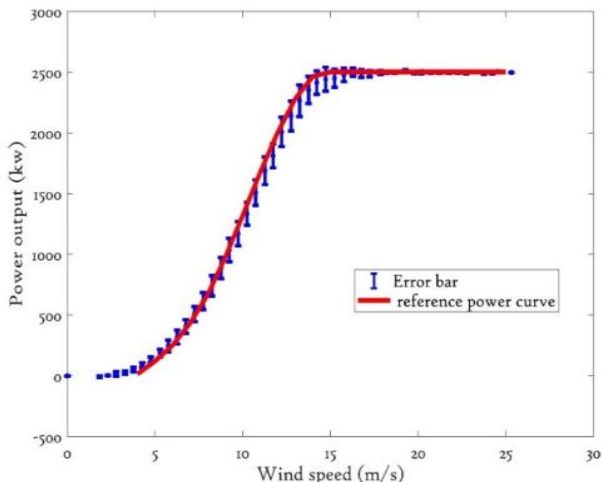

Figure 7: Binned power curve with error bars

Once the reference power curve has been constructed, the next step is to assess potentially unhealthy incoming data point by point against the relevant bin and its uncertainly by probabilistic assessment. In the probabilistic binning method, Fisher's combined probability test (describe in 6.1.1) is used to multiply two sequential $\mathrm{p}$ values. After testing and validation various different numbers of $p$-values it was found that the $2 \mathrm{p}$-values gives the most effective performance, and hence will be used here. Two sequential values have been used here, but this could be extended if required to give greater confidence that the data points are anomalous. A threshold of 0.005 (or significance level) is set in order to determine the aggregate number of anomalous bin values. A graph of yaw error together with alarm detection based on the probabilistic approach is plotted in figure 8. From the graph, anomalous performance due to yaw misalignment or a yaw drive control issue identified. This is further confirmed by figure 9, where it can be seen that the nacelle is stuck in a fixed position for an extended period of time.

Using this method, the first alarm recorded at 00:50 15-04-2009, approximately 4 hours after the yaw fault was first identified (in figure 8) at 21: 00 14-04-2009. It should be noted that this time series also indicates a limited number of false alarms. Overall though, the proposed approach, based on the probabilistic binned power curve approach, allows identification of this significant performance issue, and does this relatively quickly.

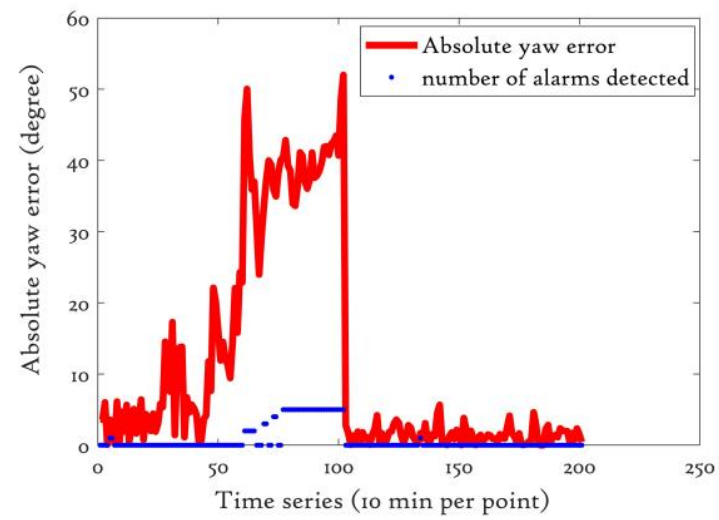

Figure 8: Yaw error detection using probabilistic assessment of binned power curve.

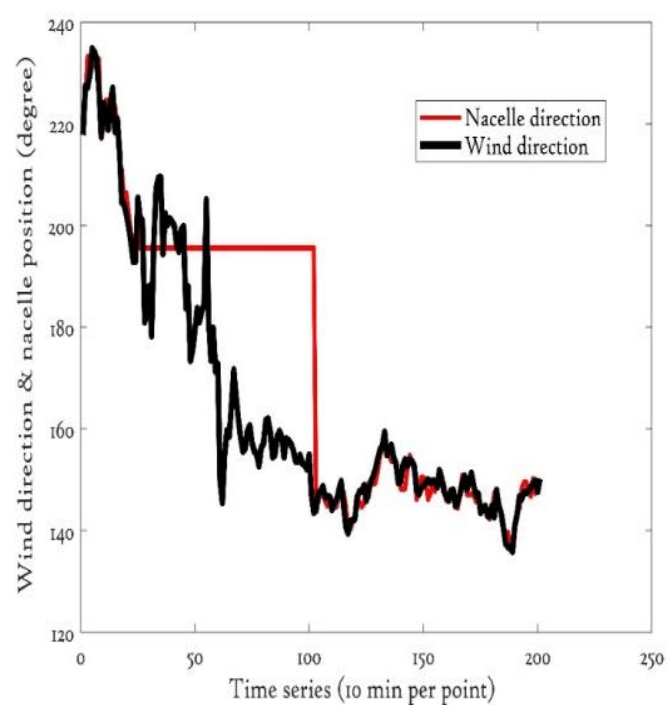

Figure 9: Time series of wind direction and nacelle position

\subsubsection{Probabilistic assessment of incoming data using a real time power curve}

Wang and Infield, [47], proposed an approach to anomaly detection by comparing a so called real-time power curve with a reference power curve using Welch's hypothesis test with a confidence interval of $99.5 \%$ i.e. a significance level of 0.005 . Selection of this provisional figure was on intuitive basis where the calculated likelihood is not unreasonable. A significance level of 0.005 was used to determine the aggregate number of anomalous bin values at each time point for an entire power curve. These are plotted as a time series as shown in figure 10 and record the anomalous performance due to the yaw drive or yaw drive control fault.

Using this algorithm, an alarm would have been raised at $03: 00$ on 15/04/2009, 6 hours after the fault occurs (at 21:00 on $14 / 04 / 2009)$.

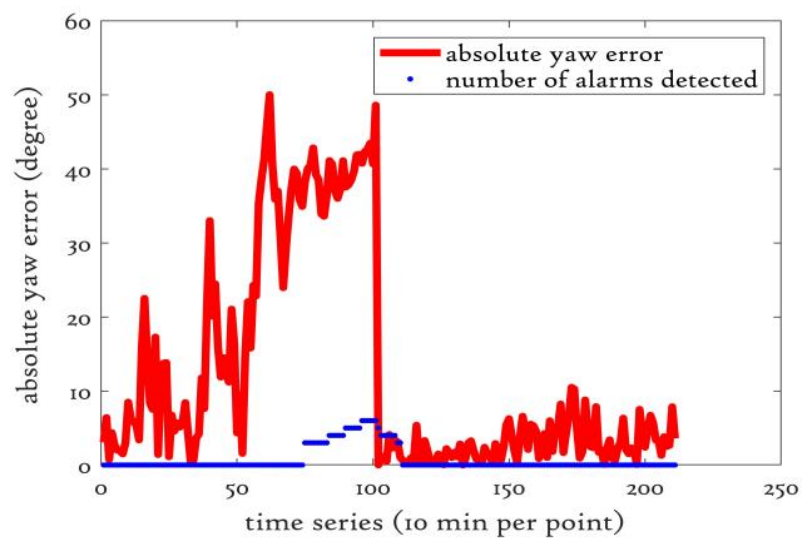

Figure 10: Absolute yaw error detection using online power curve model

\subsubsection{Probabilistic assessment of incoming data using a Gaussian Process to represent the power curve}


This paper is a post-print of a paper submitted to and accepted for publication in IET Renewable Power Generation and is subject to Institution of Engineering and Technology. The copy of record is available at IET Digital Library.

A GP power curve model intrinsically represents fitting errors and thus model accuracy as describe in section 5. An assessment of a GP power curve can be done using confidence intervals (CI). These GP confidence intervals provide information on the uncertainty surrounding an estimation, but are themselves model based estimates, [45]. Data points that lie outside of the confidence intervals can be considered anomalous, signifying a potential malfunction of the wind turbine. In order to make an accurate uncertainty estimate, it is desirable to modify the confidence intervals, as describe in our previous work. Modified confidence intervals together with a fitted GP power curve is shown in figure 11. It is worth noting that figure 11 is a fitted GP power curve with CI obtained from a healthy operational turbine using the model describe in section 5; this will be used as the reference GP power curve.

A confidence interval (CI) of $95 \%$ reflects a significance level of 0.05 and was used in the GP model to determine the sequential anomalous data point values at each time for an entire reference GP power curve. The incoming data points are assessed on a point by point basis against the reference GP power curve and probabilistic assessment undertaken. The Fisher test as describe in section 6.1.1 was used to combine 3 p-values with a threshold of 0.008 applied to filter the individual p-values. False alarms cause additional operational costs and so it is desirable to construct an effective GP model that generates no false alarms. To achieve this, the GP is model adjusted by changing the probability threshold until no false alarms occur; a threshold of 0.008 gives accurate results with no false positives, and hence is used here.

Figure 12 shows the effectiveness of the GP algorithm where the aggregate number of anomalous values at each time point are plotted as a time series together with absolute yaw error.

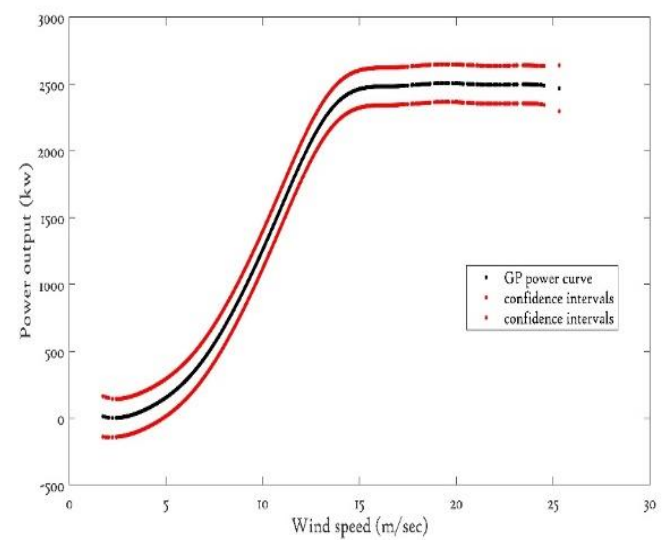

Figure 11: Reference power curve fitting with confidence intervals using GP

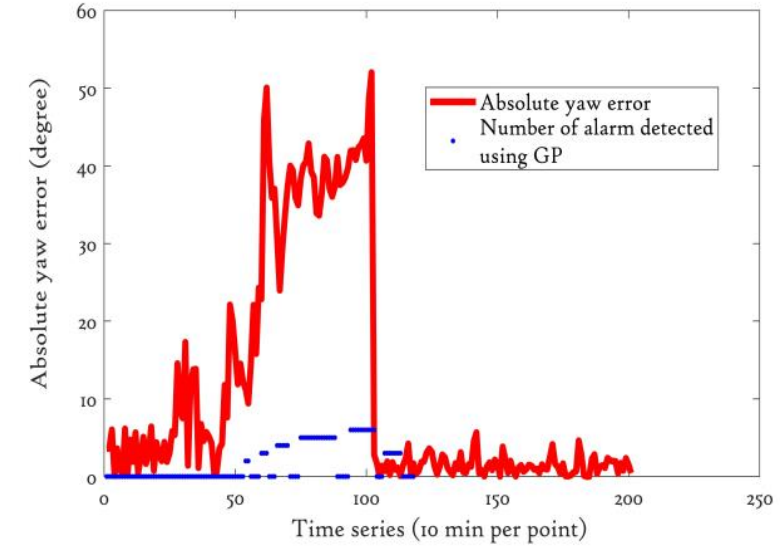

Figure 12: Absolute yaw error detection using GP model

Using the GP algorithm, an alarm would have been raised at 22:30 on 14/04/2009, just 1.5 hrs after the start of the yaw fault at 21:00 on 14/04/2009, as shown in figure 12 . Moreover, as described above, there are no false positives, confirming that the GP approach provides both fast and robust fault identification.

\section{Comparison of performance of the different methodologies}

The performance of the three different approaches to detection of a power performance anomaly associated with a yaw control fault have been assessed in section 6 above. In this section the performance of three different techniques are compared, mainly in terms of speed of detection. Speed of detection is important since continued operation, in this case with large yaw angle errors, will lead to enhanced damage rates. The response of the three models are brought together in figure 13. As can be identified from the nacelle position time series (figure 9), the yawing fault started at 21:00 on $14 / 04 / 2009$ and hence this will be used as the benchmark to judge the capability each of the models' capability to rapidly detect the fault. By doing comparative analysis, it has been found that GP approach is able to detect yaw error earlier, just 1.5 hours after the fault occurs, while probabilistic binning approach took around 4 hours, and the real-time power curve algorithm took 6 hours to identify the fault, as shown in figure 13 and in table 2 . Not only is the GP method able to detect the yaw misalignment quickly, but it produces no false positives, in contrast to the probabilistic power curve. 
This paper is a post-print of a paper submitted to and accepted for publication in IET Renewable Power Generation and is subject to Institution of Engineering and Technology. The copy of record is available at IET Digital Library.

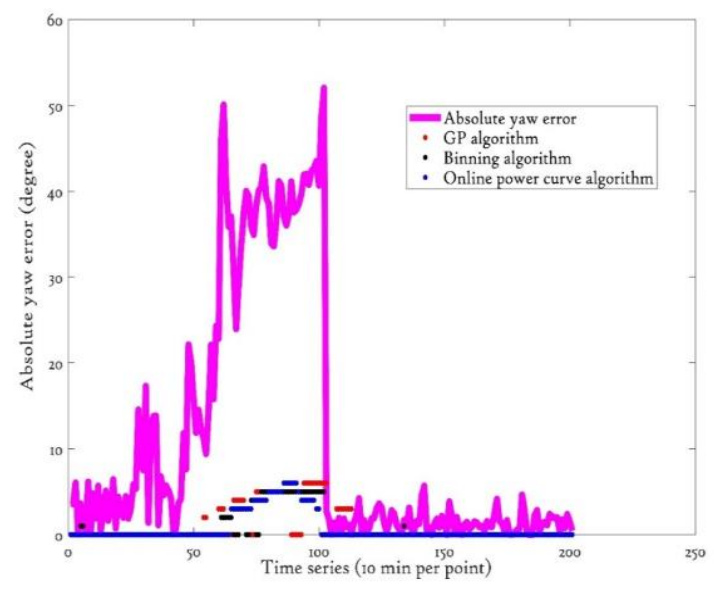

Figure 13: Comparative analysis of different models for absolute yaw error detection

Table 2: Alarm record and detection by each approach

\section{Conclusions and discussions}

Gaussian Process (GP) models have been shown to provide an effective approach to condition monitoring. Models based on GP and binning have been analyzed to assess their effectiveness in terms of capability to detect in advance (and by how much) anomalous performance related to yaw misalignment. Accurate modeling of the reference power curve is vital in the development of algorithms to detect such anomalies. It has been found that the proposed GP model is able to detect anomalies at 22:30 on 14/04/2009, just $1.5 \mathrm{hrs}$. after the fault, considerably more quickly than the two other methods investigated. Moreover, so this example, the GP approach produced no false positives.

While dealing with GPs it is important to note the data management challenge. Due to the inverse cubic problem, [48], it is not desirable to include large number of SCADA data points in the models, either for training or for fitting. Some methods based on state-space algorithms, [49,50], can help with this inverse cubic problem but these still requires high processing power and computational cost in dealing with large SCADA datasets of the sort encountered in wind turbine power curve fitting. Hence striking the balance between these two is vital for GP model accuracy.

Future work will test the approach developed here against a wide range of different turbine faults.

\section{Acknowledgments}

This project has received funding from the European Union's Horizon 2020 research and innovation programme under the Marie Sklodowska-Curie grant agreement No 642108.

\section{References}

[1] IEC 61400-12-1: 'International Electro-Technical Commission, Wind Turbines — Part 12-1: Power performance measurements of electricity producing wind turbines',2016
[2] Clara M. St. Martin, Julie K. Lundquist, Andrew Clifton, Gregory S. Poulos, and Scott J. Schreck. Wind turbine power production and annual energy production depend on atmospheric stability and turbulence. Wind Energy. Sci. Manuscript under review; published: 17 June 2016

[3] Emil Hedevang. Wind turbine power curves incorporating turbulence intensity. Wind Energy 2014; 17:173-195

[4] V. Bulaevskaya, S. Wharton, A. Clifton, G. Qualley, and W. O. Miller. Wind power curve modeling in simple and complex terrain using statistical models. J. Renewable Sustainable Energy (2015)

[5] Huan Long, Long Wang, Zijun Zhang, Zhe Song, and Jia Xu. Data-Driven Wind Turbine Power Generation Performance Monitoring. IEEE Trans. Ind. Electronics, Vol. 62, No. 10, Oct. 2015. pp 6627-6635

[6] Onder Uluyol, Girija Parthasarathy, Wendy Foslien, and Kyusung Kim. Power Curve Analytic for Wind Turbine Performance Monitoring and Prognostics. Annual Conference of the Prognostics and Health Management

\begin{tabular}{ccc}
\hline Model & $\begin{array}{c}\text { Alarm } \\
\text { detected }\end{array}$ & $\begin{array}{c}\text { Time taken to } \\
\text { identify the fault }\end{array}$ \\
\hline Online power curve & $3: 00$ on & 6 hours \\
model & $15 / 4 / 2009$ & \\
\hline Probabilistic & $00: 50$ on & $\sim 4$ hours \\
assessment using & $15 / 4 / 2009$ & \\
binning & \\
\hline Probabilistic & $22: 30$ on & 1.5 hours \\
assessment using & $14 / 4 / 2009$ & \\
GP &
\end{tabular}

Society, Montreal, Canada, Sept. 2011

[7] M. Lydiaa, S. Suresh Kumarb, A. Immanuel Selvakumara and G. Edwin Prem Kumar. A comprehensive review on wind turbine power curve modeling techniques. Renewable and Sustainable Energy Reviews 30 (2014) 452-460

[8] C. Carrillo n, A.F. Obando Montaño, J. Cidra's, E. Di'az-Dorado. Review of power curve modelling for wind turbines. Renewable and Sustainable Energy Reviews 21 (2013) 572-581

[9] Shahab Shokrzadeh, Mohammad Jafari Jozani, and Eric Bibeau. Wind Turbine Power Curve Modeling Using Advanced Parametric and Nonparametric Methods. IEEE Trans. Sust. Energy, Vol. 5, No. 4, Oct. 2014, pp 1262-1269

[10] Georgios Alexandros Skrimpas, Christian Walsted Sweeney, Kun S. Marhadi, Bogi Bech Jensen, Nenad Mijatovic and Joachim Holbøll. Employment of Kernel Methods on Wind Turbine Power Performance Assessment. IEEE Trans. Sust. Energy, Vol. 6, No. 3, July 2015, pp 698-706

[11] Georgios Alexandros Skrimpas, Christian Walsted Sweeney, Kun S. Marhadi, Bogi Bech Jensen, Nenad Mijatovic, and Joachim Holbøll. Detection of Wind Turbine Power Performance Abnormalities Using 
This paper is a post-print of a paper submitted to and accepted for publication in IET Renewable Power Generation and is subject to Institution of Engineering and Technology. The copy of record is available at IET Digital Library.

Eigenvalue Analysis. Annual Conference of the Prognostics and Health Management Society, 2014

[12] Giwhyun Lee, Yu Ding, Le Xie and Marc G. Genton. A kernel plus method for quantifying wind turbine performance upgrades. Wind Energy 2015; 18:12071219

[13] Giwhyun Lee, Yu Ding, Marc G. Genton, and Le Xie. Power Curve Estimation with Multivariate Environmental Factors for Inland and Offshore Wind Farms. Journal of the American Statistical Association, 110:509, March 2015, pp 56-67

[14] Rodrigo J. de Andrade Vieira, Miguel A. Sanz-Bobi and So Kato. Wind Turbine Condition Assessment based on Changes Observed in its Power Curve. Proceeding of International Conference on Renewable Energy Research and Applications Madrid, Spain, 20-23 October 2013, pp 31-36

[15] M S Mohan Raj, M Alexander and M Lydia. Modeling of Wind Turbine Power Curve. Proceedings of 2011 IEEE PES Innovative Smart Grid Technologies conference, India

[16] Simon Gill, Bruce Stephen, and Stuart Galloway. Wind Turbine Condition Assessment Through Power Curve Copula Modeling. IEEE Trans. Sust. Energy, Vol. 3, No. 1, Jan. 2012, pp 94-101

[17] Wang, Y., Infield, D., Stephen, B. \& Galloway, S. Copula based model for wind turbine power curve outlier rejection; Wind Energy, Vol.17, Issue 11, pp 1677-1688, Nov. 2014

[18] Jin Zhou, Peng Guo and Xue-ru Wang. Modeling of Wind Turbine Power Curve Based on Gaussian Process. Proceedings of the 2014 International Conference on Machine Learning and Cybernetics, Lanzhou, 13-16 July, 2014, pp 71-76

[19] V. Bulaevskaya, S. Wharton, A. Clifton, G. Qualley, and W. O. Miller. Wind power curve modeling in complex terrain using statistical models. J. Renewable Sustainable Energy 7, 013103 (2015)

[20] 'Renewable energy sources :wind', http://www.beaufortcourt.com/energy-generation/windenergy, accessed 27 November 2017

[21] 'Flexible solutions to optimize turbine performance', http://www.aventlidartechnology.com/en/applications/y aw-error-correction 116.html, accessed 15th December 2018

[22] 'Optimising annual energy production with apt handling of yaw misalignment' 4th Wind Operations and Maintenance Canada 2017. $4^{\text {th }}$ November,2013

[23] Kragh, K. A. and Hansen, M. H. (2014). Load alleviation of wind turbines by yaw misalignment. Wind Energy., 17: 971-982

[24] 'Wind turbines: correcting yaw error', http://www.engineerlive.com/content/wind-turbinescorrecting-yaw-error, accessed 19th December 2018

[25] 'How to improve the LCOE of wind power', http://new.abb.com/power-generation/in-control/012015/how-to-improve-the-lcoe-of-wind-power, publish in 2015

[26] Mamidipudi, P., Dakin, E., Hopkins, A. F., Belen, C., Leishman, J. G. Yaw Control: The Forgotten Controls Problem. EWEA Brussels, 2011
[27] M. Schlechtingen, I.F. Santos Comparative analysis of neural network and regression based condition monitoring approaches for wind turbine fault detection Mechanical Systems and Signal Processing, 25 (5) (2011), pp. 1849-1875

[28] M. Schlechtingen, I. F. Santos, and S. Achiche. Using datamining approaches for wind turbine power curve monitoring: a comparative study. IEEE Transactions on Sustainable Energy,vol. 4, no. 3, pp. 671-679, 2013

[29] S. Mathew. Wind Energy-Fundamentals, Resource Analysis and Economics. Springer, Berlin, Germany, 2006

[30] F. A. L. Jowder. Wind power analysis and site matching of wind turbine generators in Kingdom of Bahrain. Applied Energy, vol. 86, no. 4, pp. 538-545, 2009

[31] W. R. Powell. An analytical expression for the average output power of a wind machine. Solar Energy, vol. 26, no. 1 , pp. 77-80, 1981

[32] T.-P. Chang, F.-J. Liu, H.-H. Ko, S.-P. Cheng, L.-C. Sun, and S.- C. Kuo. Comparative analysis on power curve models of wind turbine generator in estimating capacity factor. Energy, vol. 73, pp. 88-95, 2014

[33] A. Kusiak, H. Zheng, and Z. Song. Models for monitoring wind farm power. Renewable Energy, vol. 34 no. 3, pp. 583-590,2009

[34] J.-Y. Park, J.-K. Lee, K.-Y. Oh, and J.-S. Lee. Development of a novel power curve monitoring method for wind turbines and its field tests. IEEE Transactions on Energy Conversion, vol. 29, no. 1, pp. 119-128, 2014

[35] Wind Turbines-Part 12-1: Power Performance Measurements of Electricity Producing Wind Turbines, British Standard, IEC 61400-12-1, 2006

[36] Ping Li, Song can Chen. A review on Gaussian Process Latent Variable Models. CAAI Transactions on Intelligence Technology (2016)

[37] C. E. Rasmussen \& C. K. I. Williams. Gaussian Processes for Machine Learning. the MIT Press, 2006, ISBN 026218253X

[38] Wagner, R. Antoniou, I. Pedersen, S. M. Courtney, M. S. and Jørgensen, H. E. (2009). The Influence of the Wind Speed Profile on Wind Turbine Performance Measurements. Wind Energy, Vol. 12, Issue 4, pp. 348362

[39] Fisher, R.A., 1932. Statistical Methods for Research Workers. Oliver and Boyd, London

[40] Ori Davidov. Combining p-values using order-based methods. Computational Statistics \& Data Analysis, Volume 55, Issue 7, 2011, pp. 2433-2444

[41] Brown, Morton B. 400: A Method for Combining NonIndependent, One-Sided Tests of Significance. Biometrics, vol. 31, no. 4, 1975, pp. 987992. JSTOR, JSTOR, www.jstor.org/stable/2529826

[42] James T Kost, Michael P McDermott. Combining dependent P-values. Statistics \& Probability Letters Volume 60, Issue 2, 15 November 2002, Pages 183-190.

[43] McDermott, M.P., 1999. Generalized orthogonal contrast tests for homogeneity of ordered means. Canad. J. Statist. 27,457-470

[44] Cox, C.P., Han, C., 1982. Testing multivariate means when the covariance matrix has intraclass correlation structure. J. Statist. Comput. Simulation 16, 97-107 
This paper is a post-print of a paper submitted to and accepted for publication in IET Renewable Power Generation and is subject to Institution of Engineering and Technology. The copy of record is available at IET Digital Library.

[45] Neyman, J. (1937). Outline of a Theory of Statistical Estimation Based on the Classical Theory of Probability. Philosophical Transactions of the Royal Society A. 236: 333-380. doi:10.1098/rsta.1937.0005

[46] R. K. Pandit and D. Infield. Using Gaussian process theory for wind turbine power curve analysis with emphasis on the confidence intervals. 2017 6th International Conference on Clean Electrical Power (ICCEP), Santa Margherita Ligure, 2017, pp. 744-749. doi: 10.1109/ICCEP.2017.8004774

[47] Yue Wang, David G. Infield. Power Curve Based Online Condition Monitoring for Wind Turbines. COMDEM 2013 conference. doi: 10.13140/2.1.4492.9928

[48] Joseph Gonzalez. Linear-Time Inverse Covariance Matrix Estimation in Gaussian Processes. http://www.cs.cmu.edu/ sahong/docs/jegonzal_sahong final.pdf 